\title{
Efectos del contenido funcional de la instrucción sobre el desempeño en igualación a la muestra de segundo orden
}

\author{
Carlos Wilcen Villamil Barriga ${ }^{1 *}$, Telmo Eduardo Peña-Correal ${ }^{2}$ y Luis Alberto Quiroga-Baquero ${ }^{3}$ \\ ${ }^{1}$ Universidad de Guadalajara - CEIC, ${ }^{2}$ Pontificia Universidad Javeriana, ${ }^{3}$ Universidad Santo Tomás
}

Recibido, octubre 19/2015

Concepto de evaluación, abril 26/2016

Aceptado, junio 12/2016

\begin{abstract}
Referencia: Villamil, C.W., Peña-Correal, T.E. \& Quiroga-Baquero, L.A. (2018). Efectos del contenido funcional de la instrucción sobre el desempeño en igualación a la muestra de segundo orden. Acta colombiana de Psicologia, 21(1), 44-56. doi: http://www.dx.doi.org/10.14718/ ACP.2018.21.1.3
\end{abstract}

Resumen

En el presente estudio se evaluó el efecto del contenido funcional de la instrucción sobre la adquisición, transferencia y descripción de la ejecución en una tarea de igualación a la muestra de segundo orden. Se asignaron veinte estudiantes de psicología a uno de cuatro grupos experimentales: (a) instrucción procedimental al inicio de la tarea (PInicio); (b) instrucción procedimental en cada ensayo (PEnsayo); (c) instrucción referida a instancias en cada ensayo (IEnsayo); y (d) instrucción referida a relación en cada ensayo (REnsayo). Se analizó la proporción de aciertos, la cual fue superior en los grupos IEnsayo y REnsayo en la fase de entrenamiento, para los grupos PInicio, PEnsayo y REnsayo en pruebas de transferencia extrainstancia y extramodal, y para el grupo IEnsayo en pruebas de transferencia extrarelacional; la mayor cantidad de descripciones adecuadas de ejecución se presentó en el grupo REnsayo. Estos resultados muestran que el control abstracto del estímulo se puede presentar tanto por transferencia de función a través de instrucciones como por diferenciación de casos positivos y negativos de las contingencias, lo cual se ve potenciado por la presencia concurrente de la instrucción y el arreglo estimulativo.

Palabras clave: Igualación a la muestra, control abstracto de estímulo, contenido instruccional, función instruccional, función dimensional.

\section{Effects of the functional content of instructions on second-order matching-to-sample performance}

\begin{abstract}
The effect of the functional content of instructions on the acquisition, transfer and description of performance in a second order matching-to-sample task was evaluated. Twenty undergraduate students were assigned to one of four experimental groups: (a) procedural instruction at the start of the task (PStart); (b) procedural instruction in each trial (PTrial); (c) instruction referring to instances in each trial (ITrial); and (d) instruction referring to relation in each trial (RTrial). The proportion of correct attempts was analyzed, and was found to be higher in the ITrial and RTrial groups for the training phase, and for the PStart, PTrial and RTrial groups in the extra-instance and extra-modal transfer test, as well as for the ITrial group in the extra-relational test; the larger number of accurate performance descriptions was observed in the RTrial group. These results show that abstract stimulus control can be presented either by way of transfer of functions throughout instructions, or by differentiation of positive and negative cases of the contingencies, which is enhanced by the concurrent presence of the instruction and the stimulus array.

Key words: Matching-to-sample, abstract stimulus control, instructional content, instructional function, dimensional function.
\end{abstract}

\footnotetext{
Centro de Estudios e Investigaciones en Comportamiento, Universidad de Guadalajara, Calle Francisco de Quevedo \# 180, Col. Arcos Vallarta, CP. 44130, Guadalajara, Jalisco, México.wilcenv@gmail.com
} 


\title{
Efeitos do conteúdo funcional da instruçáo sobre o desempenho em igualaçáo à amostra de segunda ordem
}

\begin{abstract}
Resumo
Neste estudo, avaliou-se o efeito do conteúdo funcional da instrução sobre a aquisição, transferência e descrição da execução numa tarefa de igualação à amostra de segunda ordem. Foram designados vinte estudantes de psicologia a um de quatro grupos experimentais: (a) instrução procedimental ao início da tarefa (PInício); (b) instrução procedimental em cada ensaio (PEnsaio); (c) instrução referida a instâncias em cada ensaio (IEnsaio), e (d) instrução referida à relação em cada ensaio (REnsaio). Analisou-se a proporção de acertos, a qual foi superior nos grupos IEnsaio e REnsaio na fase de treinamento, para os grupos PInício, PEnsaio e REnsaio em testes de transferência extrainstância e extramodal, e para o grupo IEnsaio em testes de transferência extrarrelacional; a maior quantidade de descrições adequadas de execução se apresentou no grupo REnsaio. Esses resultados mostram que o controle abstrato do estímulo pode ser apresentado tanto por transferência de função por meio de instruções quanto por diferenciação de casos positivos e negativos das contingências, o que se vê potencializado pela presença simultânea da instrução e do acordo estimulativo.

Palavras-chave: Conteúdo instrucional, controle abstrato de estímulo, igualação à amostra, função dimensional, função instrucional.
\end{abstract}

\section{INTRODUCCIÓN}

El estudio de las relaciones funcionales entre el comportamiento de los organismos y los objetos y eventos del ambiente se ha fundamentado en la segmentación de al menos dos eventos discretos, a saber, los de estímulo y los de respuesta (Baum, 2013). Dicha segmentación ha permitido que se propongan diversos eventos de estímulo antecedentes a los eventos de respuesta en términos de sus propiedades funcionales, tales como los estímulos incondicionales, condicionales, elicitantes, evocativos, excitatorios, inhibitorios, discriminativos, instruccionales y dimensionales, entre otros (Ribes, 1997).

Se asume que estas funciones de estímulo ejercen control sobre las propiedades de la conducta en términos de las relaciones de condicionalidad pavlovianas u operantes, lo que ha dado lugar al campo de estudio conocido como control de la conducta por los estímulos (Dinsmoor, 1995a, 1995b). En el caso de las contingencias operantes con animales no humanos se han descrito tres formas de control antecedente: la discriminación simple, la discriminación condicional y el control abstracto de estímulos (Harrison, 1991; Mackay, 1991); mientras que para el estudio con humanos se ha formulado, adicionalmente, el control por estímulos verbales antecedentes (Hayes, 1989).

En la discriminación simple se identifican dos tipos de estímulo antecedente: los estímulos discriminativos $\left(\mathrm{E}^{\mathrm{D}}\right)$ y los estímulos delta $\left(\mathrm{E}^{\Delta}\right)$; los cuales, respectivamente, determinan la ocasión en la cual la respuesta es o no reforzada (Dinsmoor, 1995a). Por otra parte, en la discriminación condicional las funciones $\mathrm{E}^{\mathrm{D}}$ y $\mathrm{E}^{\Delta}$ cambian dependiendo de la ocurrencia de otro evento denominado estímulo condicional
$\left(\mathrm{E}^{\mathrm{C}}\right)$ (Mackay, 1991). Es preciso resaltar que en la discriminación simple las funciones $\mathrm{E}^{\mathrm{D}} \mathrm{y} \mathrm{E}^{\Delta}$ se mantienen constantes o son absolutas en su relación con el reforzamiento de la respuesta, mientras que en la discriminación condicional las funciones $\mathrm{E}^{\mathrm{D}}$ y $\mathrm{E}^{\Delta}$ se caracterizan por ser variables $\mathrm{o}$ relativas en su relación con el estímulo condicional y el reforzamiento de la respuesta (Saunders \& Williams, 1998).

Esta función del estímulo condicional coincide con lo que Goldiamond (1966) denominó como función instruccional $\left(\mathrm{E}^{\mathrm{Di}} \mathrm{y} \mathrm{E}^{\Delta \mathrm{i}}\right.$ ), así como las funciones discriminativa y delta coinciden con la denominada función dimensional $\left(\mathrm{E}^{\mathrm{D}} \mathrm{y} \mathrm{E}^{\Delta}\right.$, manteniendo la misma nomenclatura). Según Goldiamond (1966), la función $\mathrm{E}^{\mathrm{Di}}$ se caracteriza por restringir las alternativas de respuesta a las modalidades en que los eventos de estímulo presentan la función $\mathrm{E}^{\mathrm{D}}$ dimensional; en otras palabras, la función instruccional determina el control $\mathrm{E}^{\mathrm{D}}$ o $\mathrm{E}^{\Delta}$ que puede ejercer una propiedad de los estímulos.

Adicionalmente, si las funciones $\mathrm{E}^{\mathrm{Di}} \mathrm{y} \mathrm{E}^{\mathrm{D}}$ sostienen una relación constante a través de propiedades variantes de los objetos, el responder se caracteriza como una discriminación relacional, y el control que ejercen tales estímulos se denomina control abstracto del estímulo (Goldiamond, 1966; Ribes, 2000). Por ejemplo, si el reforzamiento ante una propiedad constante (e.g., forma triangular) ocurre en condiciones de variación de otras propiedades (e.g., posición, color, tamaño, textura, etc.) es posible afirmar que la respuesta a dicha propiedad constante muestra control instruccional y que la respuesta a un elemento particular (e.g., un triángulo-amarillo-grande) muestra control dimensional, lo cual es sinónimo de abstracción, conceptualización, o comportamiento conforme a una regla 
(Goldiamond, 1966); así, se dice que se ha abstraído la regla responda al triángulo ${ }^{1}$.

Por último, en el caso de la conducta humana se han planteado relaciones de control por estímulos verbales antecedentes cuya función se tipifica como regla. La relación de control se ha denominado en general como conducta gobernada por reglas (Vaughan, 1989) o, en particular, como control instruccional (Baron \& Galizio, 1983), la cual implica la correspondencia entre el contenido de una descripción que especifica o refiere las relaciones de dependencia entre el comportamiento efectivo y los eventos en una situación y la conducta que se emite en dicha situación (Martínez, Ortiz \& González, 2002; Ortiz, González \& Rosas, 2008; Ortiz, González, Rosas \& Alcaraz, 2006). De esta forma, una regla puede describir relaciones que impliquen control de estímulo simple, condicional o abstracto.

Ahora bien, se suele considerar que el proceso por excelencia mediante el cual se establece el control discriminativo es el reforzamiento diferencial. La presentación u omisión del reforzador ante la respuesta que ocurre frente a cambios de estímulo en la discriminación simple, o frente a relaciones estímulo-estímulo en la discriminación condicional, permite mayor diferenciación de las contingencias positivas y negativas implicadas en las funciones $\mathrm{E}^{\mathrm{D}}$ o $\mathrm{E}^{\Delta}$ absolutas o relativas, respectivamente (Green \& Saunders, 1998; Harrison, 1991; Mackay, 1991; Saunders \& Williams, 1998).

Para el caso del establecimiento de control abstracto del estímulo, la presentación del reforzador ante los casos positivos de las relaciones estímulo-estímulo que sostienen alguna correspondencia (e.g., igual forma) y su omisión ante los casos negativos auspicia la diferenciación de las funciones $\mathrm{E}^{\mathrm{Di}} \mathrm{y} \mathrm{E}^{\mathrm{D}}$ como propiedades en correspondencia constante (Carrigan \& Sidman, 1992). Si tal diferenciación ha ocurrido -favorecida por la variación en las contingencias que resulta del reforzamiento diferencial- $y$, por ende, el control abstracto de estímulo ha sido establecido, su identificación requiere que la acción de responder ante una relación estímulo-estímulo constante se presente ante propiedades de estímulo cambiantes o novedosas. En estas, se identifica que el comportamiento se presenta de manera genérica y no ante relaciones específicas, ya que demandan

\footnotetext{
La función instruccional que plantea Goldiamond (1966) como propiedad constante no diferencia si los eventos que presentan dicha funcionalidad se refieren a una propiedad absoluta, una relación entre propiedades, una relación de relaciones, o un evento lingüístico que declara una regularidad.
}

una conducta abstracta no directamente entrenada ${ }^{2}$ (Carter \& Werner, 1978; Ribes, Moreno \& Martínez, 1998).

Por otro lado, se ha asumido que si el establecimiento de control abstracto de estímulo se presenta a través de estímulos verbales antecedentes, se limita el rango de respuestas potenciales en las contingencias, de tal manera que se ve afectada la diferenciación de las funciones $\mathrm{E}^{\mathrm{Di}} \mathrm{y}$ $\mathrm{E}^{\mathrm{D}}$ como propiedades que están en correspondencia constante; en otras palabras, en la medida en que el contenido de la descripción verbal especifica la conducta efectiva en la situación, entonces el contacto con las contingencias programadas se reduce a los casos positivos (Carrigan \& Sidman, 1992; De la Sancha, Guzmán-Díaz \& Serrano, 2015; Goldiamond, 1966; Ribes, 2000; Serrano, García \& López, 2009; Stewart \& McElwee, 2009).

En este contexto, los procedimientos de igualación a la muestra de primer (IMPO) y de segundo orden (IMSO) (Cumning \& Berryman, 1965; Fujita, 1983) se han mostrado propicios para el estudio de los procesos de discriminación condicional y de control abstracto (Zentall, Galizio $\&$ Critchfield, 2002), dado que las funciones $\mathrm{E}^{\mathrm{Di}}$ y $\mathrm{E}^{\mathrm{D}}$ son separadas en diferentes eventos; a saber, los estímulos de muestra y los estímulos de comparación. En el procedimiento IMPO, el control abstracto es propiciado por la variación de relaciones individuales muestra-comparación que sostienen la misma correspondencia, lo cual implica no solo el cambio de las funciones $\mathrm{E}^{\mathrm{D}} \mathrm{o} \mathrm{E}^{\Delta}$ de los estímulos de comparación relativos a la función $\mathrm{E}^{\mathrm{Di}}$ del estímulo de muestra, sino que diferentes comparaciones comparten la misma función $\mathrm{E}^{\mathrm{D}}$ y diferentes estímulos de muestra la misma función $\mathrm{E}^{\mathrm{Di}}$, si se sostiene la misma relación (Ribes, Torres, Barrera \& Ramírez, 1995). Por ejemplo, dos ensayos IMPO pueden presentar la misma correspondencia (e.g., igual forma) si se presenta un triángulo rojo como estímulo muestra, un cuadrado azul $\left(\mathrm{E}^{\Delta}\right)$ y un triángulo rojo $\left(\mathrm{E}^{\mathrm{D}}\right)$ como comparaciones, y en otro se presenta un cuadrado azul como estímulo muestra, un triángulo rojo $\left(\mathrm{E}^{\Delta}\right)$ y un cuadrado azul $\left(\mathrm{E}^{\mathrm{D}}\right)$ como comparaciones.

En el procedimiento IMSO existe otro evento de estímulo adicional con función $\mathrm{E}^{\mathrm{Di}}$ que condiciona la función $\mathrm{E}^{\mathrm{D}} \mathrm{o}$ $\mathrm{E}^{\Delta}$ de las relaciones muestra-comparación. Retomando el ejemplo anterior, bajo un estímulo adicional que prescriba la relación de igualdad en forma, se mantienen las mismas funciones $\mathrm{E}^{\mathrm{D}} \mathrm{o} \mathrm{E}^{\Delta}$ de los estímulos de comparación, mientras

\footnotetext{
2 En el caso humano, además, se ha sugerido que es posible que el individuo pueda formular una descripción de la forma de comportarse de acuerdo con las contingencias experimentadas (Skinner, 1966), aunque esto no es un efecto necesario de haber enfrentado las contingencias (Ribes, 2000).
} 
que bajo otro estímulo que prescriba la relación de diferencia en forma, tales relaciones se invierten.

En los procedimientos de igualación a la muestra, entonces, se pueden evaluar los efectos de diferentes variables sobre el control abstracto del estímulo al reconocer los procesos que afectan su desarrollo y transferencia, tal como el reforzamiento diferencial, el control por estímulos verbales antecedentes, entre otros.

Por ejemplo, Vega y Peña (2008), León (2015), QuirogaBaquero, Padilla, Ordoñez y Fonseca (2016) y RodríguezPérez, Silva-Castillo, Bautista-Castro y Peña-Correal (2015) han reportado el establecimiento de control abstracto del estímulo a través de diferentes procedimientos, a saber: (a) instrumental con retroalimentación continua; (b) acompañado por la presentación de instrucciones precisas o imprecisas; $y$ (c) diferentes tipos de modelado, comparando las ejecuciones en fases de adquisición, mantenimiento y transferencia. En general, los resultados muestran porcentajes de aciertos más altos en las fases de adquisición y transferencia ante la presentación de instrucciones precisas y de modelado experto, que ante otras condiciones de entrenamiento.

Específicamente, en lo que respecta al efecto de los estímulos antecedentes verbales sobre el control abstracto de estímulo, el contenido de las instrucciones ha asumido diversos valores en términos de la precisión-imprecisión o especificidad-generalidad de la información acerca de los estímulos antecedentes y consecuentes, las respuestas efectivas e inefectivas, las relaciones de condicionalidad entre ellos, e, incluso, acerca del procedimiento de la tarea experimental bajo el supuesto de que promueven grados diferenciales de participación de las contingencias enfrentadas, o de la instrucción, sobre el control de la conducta (Hickman, Plancarte, Moreno, Cepeda \& Arroyo, 2011; Ruiz-Castañeda \& Gómez-Becerra, 2016).

Por ejemplo, Ortiz et al. (2006, Experimento 1), Ortiz, Pacheco, Bañuelos y Plascencia (2007), y Ortiz y Cruz (2011) reportaron efectos diferenciales de la precisión del contenido instruccional en conjunción con variaciones en la densidad de la retroalimentación sobre las ejecuciones en tareas IMPO y sobre la elaboración de descripciones post-contacto. En estos estudios, la precisión de la instrucción varió en términos del contenido genérico o específico, pertinente o no pertinente, e irrelevante $o$ ausente, respecto del componente de respuesta (criterio de elección con base en modalidad y relación) en la tarea de igualación (Ortiz et al., 2008). A su vez, la retroalimentación asumió tres valores: ausente, continua o acumulada. Los principales hallazgos replicados en estos tres estudios fueron: (a) ejecuciones con porcentajes de aciertos cercanos a 0 en fases de entrenamiento y prueba bajo condiciones de instrucción genérica y sin retroalimentación; y (b) porcentajes de aciertos cercanos a $100 \%$ en fases de entrenamiento y prueba, condiciones de instrucción específica-pertinente y retroalimentación continua.

Por otro lado, Serrano, García y López (2006) evaluaron los efectos de una instrucción genérica que refería una relación no especificada, pero indicada por los estímulos de segundo orden (Grupo1) versus instrucciones que referían la modalidad (Grupo 2) o la relación (Grupo 3) sobre el desempeño en fases de entrenamiento y transferencia en una tarea IMSO. Cabe resaltar que en la fase de entrenamiento los estímulos de segundo orden se reemplazaron por la instrucción correspondiente para los grupos 2 y 3 . Los resultados mostraron que en el entrenamiento el Grupo 1 tuvo porcentajes de aciertos menores al $50 \%$, mientras que los grupos 2 y 3 tuvieron porcentajes superiores a $80 \%$. En las pruebas de transferencia, de los doce desempeños posibles, solo uno en el Grupo 2 (extradimensional) y dos en el Grupo 3 (en intramodal y extradimensional) fueron superiores al $80 \%$; los desempeños restantes, en todos los grupos, fueron inferiores a este porcentaje.

Hallazgos semejantes fueron reportados por GonzálezBecerra y Ortiz (2014) en una tarea IMPO, en la cual se presentaron instrucciones específicas pertinentes o descripciones que ejemplificaban el responder correcto/incorrecto, cuyos contenidos se referían a instancias, modalidades o relaciones. Si bien los resultados mostraron desempeños superiores al $80 \%$ en fase de entrenamiento para todas las condiciones, en las pruebas de transferencia las ejecuciones fueron deficientes, pues de las 32 ejecuciones posibles solo 5 en los grupos de modalidad, 11 en los de instancia y 13 en los de relación fueron superiores al $80 \%$ de aciertos.

En esta misma línea, Serrano, García y López (2008) presentaron instrucciones genéricas (Grupo 1), referidas a instancia (Grupo 2), a modalidad (Grupo 3) o a relación (Grupo 4). Dentro de un bloque de 27 ensayos ocurrieron de manera sucesiva las relaciones de identidad, semejanza en color y diferencia (sub-bloques de nueve ensayos por relación). En el caso del Grupo 1, la instrucción genérica se presentó al inicio del bloque de 27 ensayos, pero para los grupos 2, 3 y 4 cada instrucción precisa se presentó al inicio del sub-bloque correspondiente a cada relación. Los resultados mostraron que en el entrenamiento el $25 \%$ de los participantes del Grupo 1 y más del $75 \%$ en los grupos 2, 3 y 4 tuvieron porcentajes superiores a $80 \%$. En las pruebas de transferencia (intramodal, extramodal y extradimensional), de las 12 ejecuciones posibles, solamente dos en el Grupo 2, cinco en el Grupo 3 y tres en el Grupo 4 fueron superiores a $80 \%$.

De igual forma, Ribes y Zaragoza (2009, Experimento 1, Bloque 1) encontraron resultados semejantes para cuatro condiciones de entrenamiento: el Grupo 1 recibió instrucciones 
de relación y modalidad en cada ensayo; el Grupo 2 recibió instrucciones sobre la instancia que debía escoger en cada ensayo; y los grupos grupos 3 y 4 recibieron instrucciones genéricas al inicio del entrenamiento (con entrenamiento correctivo para el Grupo 4). En el entrenamiento todos los participantes lograron $100 \%$ de aciertos, mientras que en transferencia, los desempeños superiores al $80 \%$ fueron más frecuentes cuando la instrucción refería la relación que cuando refería la instancia; cuando la instrucción fue genérica, los desempeños fueron equivalentes a los del primer grupo solo cuando la retroalimentación fue correctiva.

Según lo anterior, los resultados no han sido conclusivos en relación con los efectos del contenido funcional de las instrucciones. En González-Becerra y Ortiz (2014) se utilizó un procedimiento IMPO y en Serrano et al. (2006) los estímulos de segundo orden no estuvieron presentes en el entrenamiento, por lo que los desempeños deficientes pudieron deberse a que no existieron componentes de estímulo que desarrollaran la función discriminativa instruccional. Esto contrasta con los buenos desempeños en transferencia de los estudios de Serrano et al. (2008) y de Ribes y Zaragoza (2009), en los cuales se utilizó el procedimiento IMSO y se vieron efectos diferenciales del contenido instruccional.

De esta manera, el hecho de que el grado de especificidad que se manipuló en las instrucciones no necesariamente limitara la transferencia del control abstracto -a pesar de restringir el contacto a los casos positivos de las contingencias sin contactar los casos negativos-cuestiona la función que se ha atribuido a las instrucciones sobre el control del responder abstracto, pues no todas las instrucciones con algún grado de precisión limitan el control abstracto del estímulo, aun cuando aquellas hayan limitado la variabilidad conductual.

Esto puede ser atribuible a que el contenido preciso de las instrucciones especificaba los aspectos funcionales de los componentes del estímulo como instancias (e.g., triángulo rojo), modalidades (e.g., forma, color) o relaciones (e.g., identidad, diferencia), que resultan efectivos para la igualación de las relaciones muestra-comparación. De este modo, las instrucciones cuyo contenido es referido a las relaciones y las modalidades efectivas pueden establecer una forma general de comportamiento aplicable a diferentes casos y sin limitarse a propiedades particulares de casos positivos, mientras que las instrucciones cuyo contenido refiere las instancias pueden establecer una forma concreta de responder a la situación inmediata.

Las instrucciones cuyo contenido refiere a las modalidades o relaciones promoverían transferencia del control abstracto en tanto que el comportamiento se vincula a una regularidad en la contingencia, mientras que aquellas referidas a instancias limitarían dicha transferencia en tanto que la conducta permanece ligada a los casos particulares de la contingencia. Por último, las instrucciones cuyo contenido preciso especifica los componentes de estímulo y de respuesta, pero no sus aspectos funcionales (genéricas), y aunque posibilitan el contacto con los casos positivos y negativos, pueden o no favorecer la transferencia. En cualquier caso, los efectos de tales contenidos pueden ser potenciados o atenuados por otras variables aún no claras.

Teniendo en cuenta lo anterior, el objetivo del presente estudio fue evaluar los efectos de tres tipos de contenido funcional de la instrucción (instancia, relación o procedimental) sobre la adquisición, transferencia y adecuación de la descripción de las contingencias experimentadas en una tarea de IMSO.

\section{MÉTODO}

\section{Participantes}

Participaron 20 estudiantes de primer semestre de Psicología de la Universidad Nacional de Colombia (10 hombres, 10 mujeres, rango de edad: 16-23 años) sin experiencia previa en tareas de igualación a la muestra. Se les proporcionó una bonificación económica no condicionada a su desempeño en la tarea por su participación.

\section{Instrumentos y escenario}

La tarea experimental fue programada en el software Macromedia Authorware 7.0 y presentada a través de un computador marca Dell con procesador Intel Pentium 4 y monitor de 17 pulgadas. El experimento se realizó en un cubículo de 2 × 3 metros, el cual contaba con buena iluminación y aislamiento de ruidos exteriores.

\section{Procedimiento}

Diseño experimental. El diseño fue univariado con cuatro grupos de cinco participantes cada uno, asignados de manera aleatoria. El Grupo 1 recibió una instrucción con contenido de tipo procedimental al inicio de la tarea experimental (PInicio), mientras que los grupos 2, 3 y 4 recibieron, en cada uno de los ensayos de entrenamiento, instrucciones con contenido referido al procedimiento (PEnsayo), a las instancias (IEnsayo) o a las relaciones (REnsayo), respectivamente. Todos los grupos se enfrentaron a una fase de adquisición consistente en entrenamiento y prueba de aprendizaje, y a una fase de transferencia consistente en tres pruebas, cada una seguida del requerimiento de un reporte escrito (véase Tabla 1). 
Tabla 1

Diseño y condiciones experimentales

\begin{tabular}{|c|c|c|c|c|c|}
\hline & Fase 1: Adquisición & & Fase 2: Transf & encia & \\
\hline Grupo & Entrenamiento con instrucción & \multirow{5}{*}{$\begin{array}{l}\text { Prueba de } \\
\text { aprendizaje }\end{array}$} & \multirow{5}{*}{\multicolumn{2}{|c|}{ Extrainstancia }} & \multirow{5}{*}{ Extrarrelacional } \\
\hline 1 & Procedimental al inicio (PInicio) & & & & \\
\hline 2 & Procedimental en cada ensayo (PEnsayo) & & & & \\
\hline 3 & Instancia en cada ensayo (IEnsayo) & & & & \\
\hline 4 & Relación en cada ensayo (REnsayo) & & & & \\
\hline
\end{tabular}

\section{Tarea experimental.}

Se empleó un procedimiento de igualación a la muestra de segundo orden (IMSO). En cada ensayo se presentaron dos objetos en la parte superior, un objeto en la parte central $\mathrm{y}$ tres objetos alineados en la parte inferior de la pantalla. Los objetos de estímulo empleados en la tarea fueron una serie de figuras geométricas con diferentes modalidades estimulativas (véase Tabla 2).

Para el Grupo 1 (PInicio) se presentó la instrucción una vez al inicio de la tarea experimental, antes del primer bloque de entrenamiento; para el Grupo 2 (PEnsayo) la instrucción fue la misma y se presentó en cada uno de los ensayos de entrenamiento; para el Grupo 3 (IEnsayo) las instrucciones variaban en cada ensayo de acuerdo con las instancias presentadas; y para el Grupo 4 (REnsayo) las instrucciones variaban en cada ensayo de acuerdo con el tipo de relación especificada por los estímulos de segundo orden (véase Tabla 3). En los grupos con instrucción ensayo a ensayo, el texto se presentó sobre los estímulos de segundo orden.

La fase de entrenamiento estuvo compuesta por un mínimo de 36 o un máximo de 126 ensayos, divididos en bloques de 18 ensayos consistentes, 9 problemas de identidad y 9 de semejanza por forma o color. En cada ensayo se proporcionó retroalimentación ante la elección de un estímulo de comparación con los textos correcto o incorrecto. Cada una de las pruebas estuvo compuesta por 24 ensayos: (a) prueba de aprendizaje, con 12 ensayos de identidad por color y forma y 12 de semejanza por color o forma; (b) transferencia extra-instancia, con 12 ensayos de identidad en color y forma y 12 de semejanza por color o forma; (c) transferencia extra-modal, con 12 ensayos de identidad en textura y tamaño y 12 de semejanza por textura o tamaño; y (d) transferencia extra-relacional, con 12 ensayos de diferencia en color y forma y 12 ensayos de semejanza por color o forma. En ninguna de las pruebas se proporcionó retroalimentación ni se presentaron las instrucciones utilizadas en el entrenamiento. Al final de cada prueba de transferencia se solicitó un reporte escrito de ejecución.

\section{Procedimiento general}

Una vez que el participante llegaba al laboratorio se le informaba de manera general en qué consistía la tarea,

Tabla 2

Estímulos utilizados durante la tarea experimental

\begin{tabular}{llllll}
\hline Estímulos & Formas & Colores & Tamaños & Trama & Fase \\
\hline Segundo orden & $\begin{array}{l}\text { Cruz y } \\
\text { pentágono }\end{array}$ & $\begin{array}{l}\text { Café y } \\
\text { rosado }\end{array}$ & $3 \mathrm{~cm}$ & Ninguna & $\begin{array}{l}\text { Entrenamiento, aprendiza- } \\
\text { je y transferencias }\end{array}$ \\
\hline & $\begin{array}{l}\text { Círculo, } \\
\text { triángulo y }\end{array}$ & $\begin{array}{l}\text { Amarillo, } \\
\text { azul y rojo }\end{array}$ & $3 \mathrm{~cm}$ & Ninguna & $\begin{array}{l}\text { Entrenamiento, apren- } \\
\text { dizaje y transferencia } \\
\text { extrarelacional }\end{array}$ \\
$\begin{array}{l}\text { cuadrado } \\
\text { puración }\end{array}$ & $\begin{array}{l}\text { Rombo, } \\
\text { rectángulo y } \\
\text { trapecio }\end{array}$ & $\begin{array}{l}\text { Verde, } \\
\text { naranja y } \\
\text { morado }\end{array}$ & $3 \mathrm{~cm}$ & Ninguna. & $\begin{array}{l}\text { Transferencia extrainstan- } \\
\text { cia }\end{array}$ \\
& Círculo & Azul & $4.5 \mathrm{~cm}, 2.5 \mathrm{~cm} \mathrm{y}$ & $\begin{array}{l}\text { Cuadrícula pequeña, tablero } \\
\text { de damas grande y 70 \% }\end{array}$ & Transferencia extramodal \\
\hline
\end{tabular}


luego se sentaba frente a la pantalla del computador y se le pedía que leyera cuidadosamente en dos pantallas consecutivas la información acerca de los ejercicios que tenía que desarrollar (véase Tabla 3).

Posteriormente, el participante era expuesto a dos bloques de entrenamiento de 18 ensayos; si en el segundo bloque cumplía con un criterio de $90 \%$ o más de respuestas correctas, pasaba a la prueba de aprendizaje, y si en esta cumplía con el mismo criterio de efectividad, accedía a las pruebas de transferencia. Si no superaba el criterio de efectividad, ya fuera en entrenamiento o en prueba de aprendizaje, se exponía a otro bloque de entrenamiento, hasta un máximo de siete bloques (126 ensayos). Si el participante no lograba el criterio de efectividad en ninguno de los siete bloques, se daba por concluida su participación y no accedía a las pruebas de transferencia. Se proporcionó información con respecto al paso de la fase de entrenamiento a la fase de pruebas, y al final de cada prueba de trasferencia se les solicitó un reporte escrito de descripción de las contingencias experimentadas (véase Tabla 3 ).

Tabla 3

Instrucciones presentadas en la tarea experimental

Momento de la $\quad$ Información suministrada
tarea

\begin{tabular}{ll}
\hline & Bienvenido, gracias por participar en este estudio. Esta es una investigación sobre procesos de apren- \\
dizaje que son comunes a la mayoría de las personas. La tarea que usted va a realizar no tiene que ver \\
Bienvenida
\end{tabular}$\quad \begin{aligned} & \text { con inteligencia o personalidad sino con el proceso de solución de cierto tipo de problemas. Sus datos } \\
& \text { personales serán empleados para fines investigativos exclusivamente. }\end{aligned}$

En la pantalla aparecerán seis figuras: dos arriba, una en el centro y tres abajo. Usted debe elegir una de las figuras de abajo, haciendo clic sobre ella.

Familiarización En algunas ocasiones se le informará si su elección es o no correcta. La meta es hacer tantos aciertos Grupo 1 como sea posible. Si tiene alguna pregunta hágala en este momento pues más adelante no se le podrá suministrar información adicional.

Ahora puede comenzar. Cuando esté listo haga clic en el botón "Continuar".

En la pantalla aparecerá una disposición de seis figuras y una instrucción. Usted debe elegir una de las figuras de abajo, haciendo clic sobre ella de acuerdo con lo que diga la instrucción.

Familiarización En algunas ocasiones se le informará si su elección es o no correcta. La meta es hacer tantos aciertos grupos 2, 3 y 4 como sea posible. Si tiene alguna pregunta, hágala en este momento, pues más adelante no se le podrá suministrar información adicional.

Ahora puede comenzar. Cuando esté listo haga clic en el botón "Continuar".

$\begin{array}{ll}\begin{array}{l}\text { Entrenamiento } \\ \text { grupos } 1 \text { y } 2\end{array} & \text { En la pantalla aparecen seis figuras: dos arriba, una en el centro y tres abajo. Usted debe elegir una de las } \\ & \text { figuras de abajo haciendo clic sobre ella. }\end{array}$
grupos 1 y 2 figuras de abajo haciendo clic sobre ella.

\begin{tabular}{ll}
\hline $\begin{array}{l}\text { Entrenamiento } \\
\text { Grupo 3 }\end{array}$ & $\begin{array}{l}\text { Teniendo en cuenta que las figuras de arriba son una CRUZ CAFÉ y una CRUZ ROSADA y que la } \\
\text { figura del medio es un TRIÁNGULO ROJO, escoja la figura de abajo que es un TRIÁNGULO VERDE. }\end{array}$ \\
\hline $\begin{array}{l}\text { Entrenamiento } \\
\text { Grupo 4 }\end{array}$ & $\begin{array}{l}\text { Teniendo en cuenta que las figuras de arriba son SEMEJANTES entre sí, escoja la figura de abajo que es } \\
\text { SEMEJANTE a la figura del medio. }\end{array}$ \\
\hline $\begin{array}{l}\text { Pruebas } \\
\text { Grupo 1 }\end{array}$ & A partir de este momento NO se le dirá si su elección es correcta o no. \\
$\begin{array}{l}\text { Pruebas } \\
\text { grupos 2, 3 y } 4\end{array}$ & A partir de este momento NO aparecerá la instrucción y NO se le dirá si su elección es correcta o no. \\
\hline $\begin{array}{l}\text { Pantalla de } \\
\text { descripción de } \\
\text { contingencias }\end{array}$ & $\begin{array}{r}\text { En este momento describa la forma correcta de responder en la serie de ensayos anteriores. } \\
\text { Cuando en la parte superior de la pantalla aparecían dos figuras } \\
\text { a la figura de la mitad, y cuando en la parte superior de la pantalla aparecían dos figuras } \\
\text { seleccioné la figura_la figura de la mitad. }\end{array}$ \\
\hline
\end{tabular}




\section{RESULTADOS}

La Figura 1 muestra las ejecuciones individuales en cada uno de los bloques de entrenamiento, de prueba de aprendizaje y de transferencia en términos de proporciones de aciertos (eje izquierdo de las ordenadas) y la identificación de las relaciones en la descripción de contingencias post-prueba por cada participante (eje derecho de las ordenadas).

\section{Resultados en la fase de entrenamiento}

En relación con la proporción de aciertos obtenida por cada grupo experimental en la fase de entrenamiento, teniendo en cuenta la ejecución en los bloques necesarios

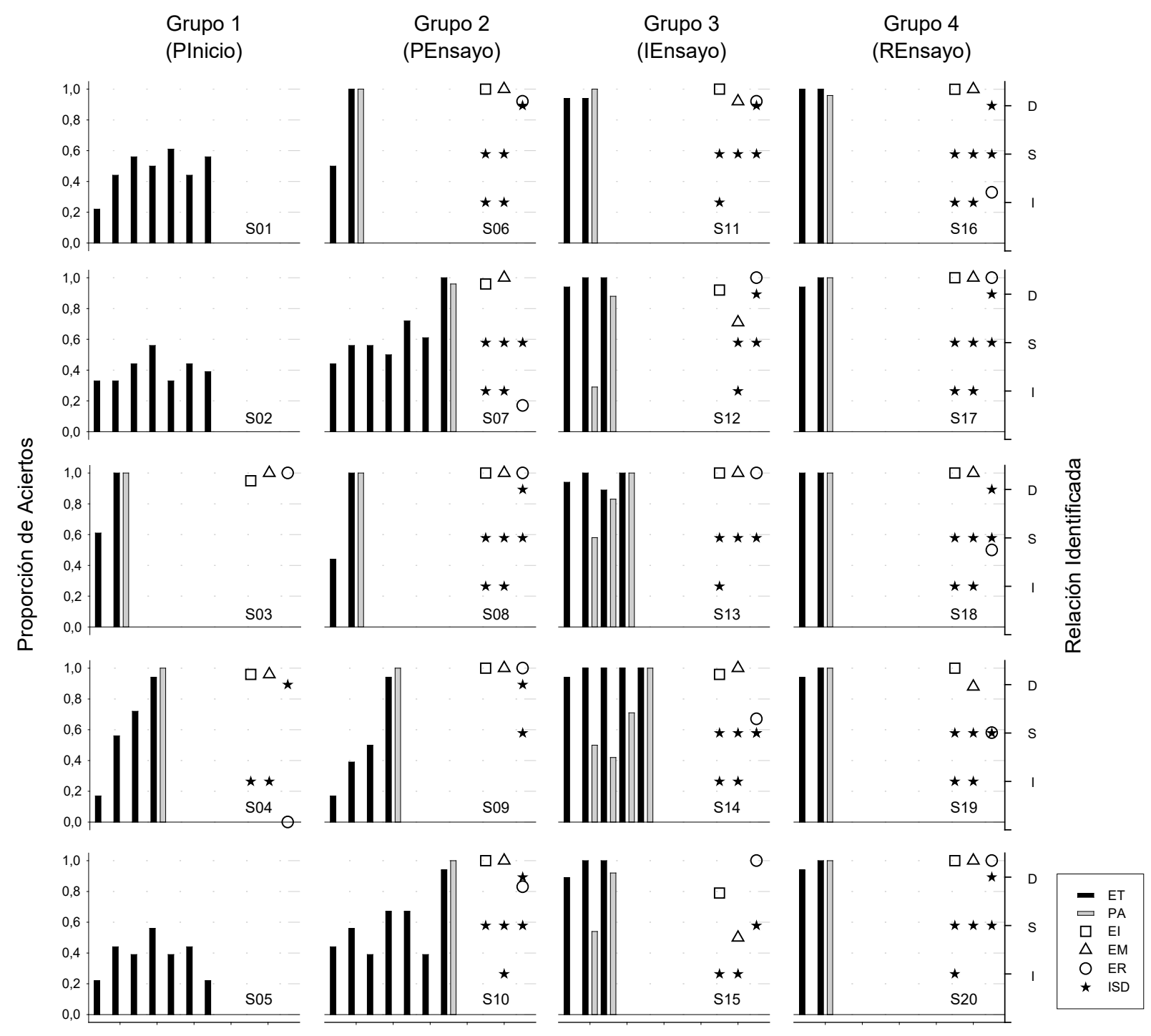

Figura 1. Proporción de aciertos y tipo de relación identificada en la descripción de contingencias. El eje izquierdo de las ordenadas representa la proporción de aciertos en entrenamiento (ET), prueba de aprendizaje (PA) y pruebas de trasferencias extrainstancia (EI), extramodal (EM) y extrarelacional (ER). En ET se presenta el número de bloques de 18 ensayos a los cuales se expuso cada participante, y en PA se presenta el número de veces que cada participante pasó por la prueba de aprendizaje. El eje derecho de las ordenadas representa mediante asteriscos las relaciones de identidad (I), semejanza (S) o diferencia (D), identificadas en las descripciones de contingencias posteriores a cada prueba de transferencia. Las descripciones en cada prueba pueden ser: adecuadas $=$ dos asteriscos; parcialmente adecuadas $=$ un asterisco; inadecuadas $=$ ningún asterisco. 
para superar el criterio, es posible identificar que en la condiciones correspondientes a los grupos PInicio y PEnsayo se encontraron los promedios de proporciones de aciertos más bajos $(M=.48 ; D . T .=.19 ; M=.61 ; D . T .=.23$, respectivamente), mientras que en las condiciones de los grupos IEnsayo y REnsayo se encontraron proporciones de aciertos más altas $(M=.96 ; D . T .=.04 ;$ y $M=.98 ; D$. $T .=.03$, respectivamente). Cabe resaltar, adicionalmente, que la variabilidad intragrupo para las condiciones PInicio y PEnsayo fue mayor que la obtenida en las condiciones IEnsayo y REnsayo.

En términos del número de ensayos de entrenamiento y prueba de aprendizaje requeridos para acceder a las pruebas de transferencia se encontró que para el Grupo PInicio el promedio fue de 89.1 ( $\mathrm{Min}=60$; Max = 126); para el Grupo PEnsayo fue de 103 (Min =60; Max =150); para el Grupo IEnsayo fue de $119(\operatorname{Min}=60 ; \operatorname{Max}=186) \mathrm{y}$, finalmente, para el Grupo REnsayo fue de 60 ( $\operatorname{Min}=60 ;$ Max $=60)$.

En relación con lo anterior, solo dos participantes del Grupo PInicio pasaron a las pruebas de transferencia, mientras que los tres participantes restantes se expusieron a siete bloques de entrenamiento consecutivos sin alcanzar el $90 \%$ de aciertos. Además, todos los participantes en el Grupo PEnsayo superaron el criterio de aprendizaje en los bloques de entrenamiento (dos de ellos requirieron siete bloques) y se expusieron a un sola prueba de aprendizaje, mientras que cuatro participantes en el Grupo IEnsayo requirieron más de tres bloques de entrenamiento y más de dos pruebas de aprendizaje para superar el $90 \%$ de aciertos. Finalmente, el Grupo REnsayo presentó las mejores ejecuciones, pues todos sus participantes superaron el criterio de efectividad en solo dos bloques de entrenamiento y uno de prueba de aprendizaje.

Estos datos permiten afirmar que la presentación de instrucciones procedimentales al inicio de las fases de entrenamiento o ensayo a ensayo promovió proporciones de aciertos más bajas en comparación con las condiciones de instrucciones referidas a instancias o a relaciones en cada ensayo. Sin embargo, cabe señalar que la condición IEnsayo promovió desempeños superiores al $90 \%$ de aciertos en los bloques de entrenamiento, pero al exponerse a las pruebas de aprendizaje (sin retroalimentación), los participantes no superaban el criterio de efectividad y debían exponerse a un nuevo bloque de entrenamiento.

\section{Resultados en las pruebas de transferencia}

Al comparar los promedios de las proporciones de aciertos en la prueba de transferencia extrainstancia se encontraron ejecuciones homogéneas, ya que en todos los grupos los promedios fueron superiores a 90 (Grupo PInicio:
$M=.96 ; D . T .=.00 ;$ PEnsayo: $M=.99 ; D . T .=.01 ;$ Grupo IEnsayo: $M=.93 ; D . T .=.08$; y Grupo REnsayo: $M=1.0$; $D . T .=.00)$, lo cual indica que la condición experimental no tuvo un efecto diferencial sobre las ejecuciones en ensayos de transferencia con instancias novedosas.

Para la prueba extramodal, el Grupo IEnsayo $(M=.82$, $D . T .=.21)$ evidenció los promedios de las proporciones de aciertos más bajos, mientras que los correspondientes a los grupos PInicio $(M=.97, D . T .=.02)$, PEnsayo $(M=1.0, D . T .=.00)$, y REnsayo $(M=.97, D . T .=.05)$ fueron cercanos a 1.0, lo cual indica que la presentación de instrucciones procedimentales al inicio o ensayo a ensayo, así como aquellas referidas a relaciones, promovieron mejores ejecuciones ante la variación de las modalidades de los estímulos.

La comparación realizada entre las medias de las proporciones de aciertos en la prueba extrarrelacional, permite identificar que el promedio del Grupo IEnsayo $(M=.91$, $D . T .=.14)$ es mayor que los correspondientes a los grupos PInicio $(M=.50, D . T .=.70)$, PEnsayo $(M=.78, D . T .=.35)$ y REnsayo $(M=.68, D . T .=.30)$. Según esto, las condiciones de instrucciones procedimentales o relacionales ensayo a ensayo auspiciaron una mayor producción de errores ante la exposición a una relación no entrenada (diferencia), pero prescrita por los estímulos de segundo orden.

\section{Identificación de relaciones en la descripción post-pruebas}

La Figura 1 (eje derecho) presenta las relaciones de identidad, semejanza o diferencia identificadas en las descripciones posteriores a las pruebas de transferencia, las cuales fueron clasificadas como adecuadas si se identificaban las dos relaciones operativas en cada prueba, parciales si se identificaba solo una relación e inadecuadas si no se identificó ninguna. De los dos participantes del Grupo 1 (PInicio) que se expusieron a pruebas de transferencia, uno proporcionó descripciones inadecuadas y el otro proporcionó tres descripciones parcialmente adecuadas. En el Grupo 2 (PEnsayo) se encontraron diez descripciones adecuadas, tres parciales y dos inadecuadas; en el Grupo 3 (IEnsayo), siete descripciones adecuadas, siete parciales y una inadecuada; y, finalmente, en el Grupo 4 (REnsayo), 13 descripciones adecuadas, dos parciales y ninguna inadecuada.

\section{DISCUSIÓN}

El presente experimento investigó el efecto de distintos contenidos instruccionales sobre el desempeño en fases de adquisición y transferencia en una tarea de IMSO y sobre la descripción de las contingencias experimentadas. Los resultados de las ejecuciones en la fase de entrenamiento 
muestran que: (a) todos los participantes expuestos a la presentación de instrucción en cada ensayo (grupos PEnsayo, IEnsayo y REnsayo) superaron el criterio de efectividad, mientras que en la condición de exposición a instrucciones al inicio del entrenamiento (Grupo PInicio), solo dos participantes lo superaron; y (b) las condiciones de exposición a instrucciones referidas a instancias (Grupo IEnsayo) y a relaciones (Grupo REnsayo) promovieron los desempeños más efectivos en fases de entrenamiento, en términos de un mayor número de aciertos y un menor número de ensayos requeridos para superar el criterio de efectividad establecido. Sin embargo, en la prueba de aprendizaje la condición REnsayo promovió desempeños cercanos a una proporción de 1.0 de aciertos, mientras que la condición IEnsayo requirió de dos o más pruebas para que los participantes superaran el criterio de efectividad.

Los resultados anteriores pueden ser considerados a la luz de los planteamientos de Goldiamond (1966) y Layng, Sota y León (2011), según los cuales en un arreglo de estímulos tipo igualación de la muestra, los estímulos de segundo orden podrían desarrollar una función instruccional en la medida en que restringen o circunscriben la respuesta ante las propiedades de los objetos que son discriminativas, de acuerdo con el requerimiento de las contingencias de reforzamiento. Estas respuestas se limitan entonces a las propiedades dimensionales compartidas por el estímulo muestra y un estímulo de comparación (color, forma, etc.), que cambian momento a momento, pero que mantienen una correspondencia constante. Según Layng et al. (2011), es posible que ciertas propiedades de un estímulo adquieran control discriminativo instruccional $\left(\mathrm{E}^{\mathrm{Di}}\right)$ por transferencia de función a través de un enunciado verbal, lo que delimita, de este modo, las propiedades discriminativas dimensionales $\left(\mathrm{E}^{\mathrm{D}}\right.$ $\mathrm{y}^{\Delta}$ ) relevantes de elección, o a través de la diferenciación resultante de la exposición a consecuencias de los casos positivos y negativos que proporciona la retroalimentación diferencial ante contingencias cambiantes.

En la condición del Grupo PInicio, el hecho de ser expuesto a una instrucción procedimental al inicio de la tarea que especificaba la cantidad y ubicación de los estímulos en la pantalla y el requerimiento de elección de uno de ellos no favoreció la emergencia de control abstracto de estímulo; es decir, ni el establecimiento de funciones $\mathrm{E}^{\mathrm{Di}}$ en los estímulos de segundo orden, ni la discriminación de las modalidades y relaciones pertinentes (Ribes et al., 1995). De este modo, el supuesto de que el responder a propiedades relacionales es promovido por la diferenciación de los casos positivos y negativos que proporciona la retroalimentación diferencial ante contingencias cambiantes (De la Sancha et al., 2015; Ribes, 2000; Serrano et al., 2009; Stewart \& McElwee, 2009) solo se ve apoyado de manera parcial, pues si bien en esta condición se promovió la variabilidad conductual, el hecho de que tres de los cinco participantes no lograran pasar a las pruebas sugiere que ese no es necesariamente el caso y que hay variables adicionales por considerar.

Es posible que el ser reactivo a la función de los segmentos de estímulo implicados en la situación de igualación de segundo orden (estímulos selectores, muestra y comparación) pudo ser favorecida por la presencia concurrente de la instrucción y el arreglo estimulativo, lo cual facilitó la discriminación perceptual de las dimensiones y relaciones relevantes. Dicha facilitación pudo consistir en un direccionamiento de las respuestas de observación hacia los segmentos de estímulo que constituían el arreglo.

Este parece ser el caso para el Grupo PEnsayo, el cual se expuso a la concurrencia instrucción-arreglo estimular en cada ensayo y evidenció múltiples repeticiones en el número de bloques necesarios para superar el criterio de efectividad, pero una vez satisfecho dicho criterio, todos los participantes superaron la prueba de aprendizaje. Estos resultados sugieren que las instrucciones concurrentes promovieron la discriminación de los segmentos de estímulo, pero que el establecimiento de las funciones $\mathrm{E}^{\mathrm{Di}}$ fue resultado de la exposición directa a las contingencias positivas y negativas programadas, con lo que se promueve el surgimiento de control abstracto del estímulo. Debe considerarse la posibilidad empírica de que las instrucciones por sí mismas o su concurrencia con el arreglo de estímulos hayan promovido tales respuestas de observación, tal como lo reportan Huziwara, Souza y Tomanari (2016) en el estudio de los movimientos oculares en tareas de igualación a la muestra.

Con relación al segundo hallazgo, el Grupo IEnsayo estuvo expuesto a instrucciones que referían las propiedades relevantes de cada estímulo presente y la especificación de escoger uno de ellos, es decir, su propiedad $\mathrm{E}^{\mathrm{D}}$ dimensional. El reforzamiento de esta correspondencia pudo promover que la conducta de elección, momento a momento, quedara bajo el control de la instancia especificada en la instrucción, lo que limitaba el establecimiento de propiedades discriminativas instruccionales en los estímulos de segundo orden, de modo que la instrucción solo fungiría como un alterador de las funciones discriminativas (Schlinger, 1993) sobre un estímulo de comparación; efecto que variaría ensayo a ensayo.

Esto se hace evidente en el hecho de que los participantes tuvieran buenas ejecuciones en los bloques de entrenamiento, pero deficientes en las pruebas de aprendizaje ( $\sin$ instrucciones y sin retroalimentación), lo cual concuerda con lo reportado por Serrano et al. (2008) y Ribes y Zaragoza (2009). No obstante, el hecho de que todos los participantes superaran el criterio de efectividad en la última prueba de aprendizaje que enfrentaron plantea el cuestionamiento de si los estímulos de segundo orden promovieron la abstracción 
por sí mismos, o de cuál fue el factor que permitió el establecimiento del control abstracto de aquellos estímulos, ya que en esta condición el contenido de la instrucción solo alteraría funciones discriminativas para ensayos concretos y no facilitaría la diferenciación de casos positivos y negativos. Es posible que el control abstracto por parte de los estímulos de segundo orden haya surgido por la sobre-exposición a la prueba de aprendizaje, en la cual se hizo saliente la variabilidad de la situación en ausencia de instrucciones concretas, pero también en ausencia de retroalimentación, lo cual sugiere la necesidad de explorar empíricamente qué factores pueden influir en el desarrollo de la función instruccional en situaciones en las que no se presentan contingencias de reforzamiento que permitan identificar los casos positivos $\mathrm{y}$ negativos de las relaciones vigentes.

Por su parte, la condición de instrucciones referidas a relaciones correspondiente al Grupo REnsayo pudo favorecer el establecimiento de funciones discriminativas instruccionales (Goldiamond, 1966; Layng et al., 2011) en los estímulos de segundo orden, lo que se hace evidente tanto en la proporción de aciertos (cercanas 1.0) como en la menor cantidad de ensayos de entrenamiento y prueba de aprendizaje, en la cual todos los participantes superaron el criterio de efectividad. Una posible explicación a esto es que el contenido de la instrucción especificaba una relación abstracta, por lo cual, además de facilitar la respuestas de observación, pudo fungir como un alterador de las funciones en los segmentos de estímulo (Schlinger, 1993) y de la relación abstracta implicada. Esto puede corresponder con lo reportado por Rodríguez-Pérez et al. (2015), quienes identificaron el establecimiento de control abstracto de estímulos en una tarea IMSO con la presentación de instrucciones precisas referidas a relaciones en cada ensayo de entrenamiento y ausencia de retroalimentación; sin embargo, aunque en la presente investigación la condición instruccional fue similar, se suministró retroalimentación en cada ensayo, lo que sugiere una indagación empírica acerca de la función de la retroalimentación exclusivamente positiva en la fase de entrenamiento, de la instrucción relacional en ausencia de retroalimentación y de la posible interacción entre estas dos variables.

Por su parte, en la fase de transferencia se encontró que en las pruebas extrainstancia y extramodal los desempeños más efectivos fueron promovidos por las condiciones de instrucciones procedimentales y de instrucciones relacionales en cada ensayo (PEnsayo y REnsayo); y que en la prueba extrarrelacional los desempeños tendieron a ser homogéneos entre condiciones experimentales. Hallazgos similares fueron reportados por Ortiz et al. (2006), Rodríguez-Pérez et al. (2015) y Serrano et al. (2008) en condiciones de retroalimentación continua, así como en la presente investigación.
Según lo propuesto por Ribes (2000) y Serrano et al. (2009), la exposición a instancias negativas y positivas de las contingencias programadas en arreglos tipo igualación de la muestra resulta ser un factor necesario para el establecimiento de control abstracto de estímulo, el cual a su vez puede verse afectado en situaciones de instrucciones precisas que limitan tal variabilidad. Las ejecuciones de los grupos IEnsayo y REnsayo no apoyan esta afirmación, sino que parecen sugerir que este tipo de instrucciones puede favorecer el establecimiento de funciones discriminativas instruccionales en los estímulos de segundo orden, lo que promueve, de este modo, el responder relacional abstracto. En concordancia con esto, las condiciones de presentación de instrucciones concurrentes a los arreglos de estímulos ensayo a ensayo (PEnsayo, IEnsayo y REnsayo) promovieron ejecuciones efectivas en las fases de aprendizaje y transferencia, evidenciando el control abstracto del estímulo.

Finalmente, la adecuación de las descripciones de contingencias solicitadas al final de cada prueba de transferencia se vio favorecida por las condiciones de instrucciones procedimentales en cada ensayo ( 10 descripciones adecuadas) y relacionales en cada ensayo (13 descripciones adecuadas). Para el primer caso se observó una correspondencia entre proporciones de aciertos superiores a .8 en todos sus participantes en las pruebas extrainstancia y extramodal, en cuatro de ellos en extrarrelacional y una mayor frecuencia de descripciones adecuadas en cada una de estas pruebas.

Este mismo hallazgo se evidenció en el Grupo 4 (REnsayo), sin embargo, en la prueba extrarrelacional las descripciones fueron principalmente adecuadas, pero tan solo dos participantes tuvieron proporciones de aciertos superiores a .8; lo cual puede apoyar el supuesto de que el establecimiento de control abstracto de estímulo no siempre corresponde con la formulación verbal adecuada del arreglo de contingencias (Ribes \& Rodríguez, 2001), así como se observa en el participante S03 del Grupo 1 (PInicio), quien obtuvo proporciones de aciertos superiores a .9 en todas las pruebas de transferencia, pero no logró elaborar descripciones adecuadas o parcialmente adecuadas.

Por su parte, en el Grupo 3 (IEnsayo) se observó una cantidad igual de descripciones adecuadas e inadecuadas (7 en cada caso), distribuidas de forma homogénea en cada prueba de transferencia. Esto sugiere que la identificación de una o dos relaciones operativas en cada prueba no se vio afectada por el tipo de variación que constituía cada prueba de transferencia (instancias, modalidades o relaciones). Cabe resaltar que este grupo tuvo múltiples exposiciones a bloques de entrenamiento y prueba en la fase de entrenamiento a diferencia del Grupo REnsayo, en el que todos sus participantes alcanzan el criterio de efectividad en tan solo dos bloques. 
Teniendo esto en cuenta, no es claro si la mayor frecuencia de descripciones adecuadas en cada una de las pruebas de transferencia pudo ser auspiciada por el tipo de contenido instruccional suministrado en la fase de entrenamiento por los altos desempeños en las pruebas de transferencia correspondientes o por la interacción entre estos dos factores y el desempeño en fases de entrenamiento, lo que se constituye en un insumo para futuras investigaciones.

\section{REFERENCIAS}

Baron, A., \& Galizio, M. (1983). Instructional control of human operant behavior. The Psychological Record, 33, 495-520.

Baum, W. (2013). What counts as behavior? The Molar Multiscale View. The Behavior Analyst, 36, 283-293.

Carrigan, P., \& Sidman, M. (1992). Conditional discrimination and equivalence relations: A theoretical analysis of control by negative stimuli. Journal of the Experimental Analysis of Behavior, 58, 183-204. doi: 10.1901/jeab.1992.58-183.

Carter, D. E., \& Werner, T. J. (1978). Complex learning and information processing by pigeons: A critical analysis. Journal of the Experimental Analysis of Behavior, 29, 565-601. doi: 10.1901/jeab.1978.29-565.

Cumminng, W., \& Berryman, R. (1965). The complex discriminated operant: Studies of matching-to-sample and related problems. En D. I. Mostofsky (Ed.), Stimulus generalization (pp. 284-330). Stanford, CA: Stanford University Press.

De la Sancha, O., Guzmán-Díaz, G., \& Serrano, M. (2015). Efectos de variar la posición espacial de los estímulos en la adquisición y transferencia de discriminaciones condicionales en humanos. Acta Colombiana de Psicología, 18, 47-54. doi: 10.14718/ACP.2015.18.1.5.

Dinsmoor, J. A. (1995a). Tutorial stimulus control: Part I. The Behavior Analyst, 18(1), 51-68.

Dinsmoor, J. A. (1995b). Tutorial stimulus control: Part II. The Behavior Analyst, 18(2), 253-269.

Fujita, K. (1983). Acquisition and transfer of a higher-order conditional discrimination performance in the Japanese monkey. Japanese Psychological Research, 25, 1-18.

Goldiamond, I. (1966). Perception, language, and conceptualization rules. En B. Kleinmuntz (Ed.), Problem solving: Research, method and theory (pp. 183-224). New York: Wiley.

González-Becerra, V., \& Ortiz G. (2014). Efectos del tipo y contenido de las descripciones pre-contacto sobre la conducta de discriminación condicional y las descripciones post-contacto. Acta Colombiana de Psicología, 17, 11-23. doi: 10.14718/ACP.2014.17.1.2.

Green, G., \& Saunders, R. (1998). Stimulus equivalence. En K. Lattal \& M. Perone (Eds.), Research Methods in Human Operant Behavior (pp. 229-262). New York: Plenum Press. doi: 10.1007/978-1-4899-1947-2.
Harrison, J. M. (1991). Stimulus control. En I. H. Iversen \& K. A. Lattal (Eds.), Experimental Analysis of Behavior (Part 1. pp. 351-399). Amsterdam: Elsevier.

Hayes, S. C. (1989). Rule-governed behavior: Cognition, contingencies, and instructional control. New York: Plenum Press.

Hickman, H., Plancarte, P., Moreno, D., Cepeda, L., \& Arroyo, R. (2011). Efecto del tipo de instrucción sobre el informe verbal y la ejecución en niños y adultos. International Journal of Psychology and Psychological Therapy, 11, 425-441.

Huziwara, E., Souza, D., \& Tomanari, G. (2016). Patterns of eye movement in matching-to-sample tasks. Psicología: Reflexão e Crítica, 29, 2-10. doi: 10.1186/s41155-0160010-3.

Layng, J., Sota, M., \& Leon, M. (2011). Thinking through text comprehension I: Foundation and guiding relations. The Behavior Analyst Today, 12, 3-11. doi: 10.1037/h0100706.

León, A. (2015). Modo de contacto: Efecto sobre la adquisición y transferencia de una discriminación condicional de segundo orden y la emergencia de reglas. Acta Colombiana de Psicología, 18(1), 25-36. doi: 10.14718/ ACP.2015.18.1.3.

Mackay, H. A. (1991). Conditional stimulus control. En I.H. Iversen y K.A. Lattal (Eds.), Experimental Analysis of Behavior (Part I, pp. 301-350). Amsterdam: Elsevier.

Martínez, H., Ortiz, G., \& González, A. (2002). Precisión instruccional, retroalimentación y eficacia: Efectos sobre el entrenamiento y transferencia en una tarea de discriminación condicional en adultos. Acta Colombiana de Psicología, 8 , 7-33.

Ortiz, G., \& Cruz, Y. (2011). El papel de la precisión instruccional y la retroalimentación en la ejecución y descripciones pos-contacto. Revista Mexicana de Análisis de la Conducta, 37, 69-87.

Ortiz, G., González, A., \& Rosas, M. (2008). Una taxonomía para el análisis de descripciones pre y post contacto con arreglos contingenciales. Acta Colombiana de Psicología, $11(1), 45-53$.

Ortiz, G., González, A., Rosas, M., \& Alcaraz, F. (2006). Efectos de la precisión instruccional y la densidad de retroalimentación sobre el seguimiento instruccional, la elaboración y transmisión de descripciones en tareas de discriminación condicional. Acta Comportamentalia, 14, 2, 103-130.

Ortiz, G., Pacheco, V., Bañuelos, I., \& Plascencia, L. (2007). Efecto del contacto con instrucciones, la especificidad e historia instruccional en la insensibilidad al cambio contingencial en tareas de igualación de la muestra de primer orden en humanos. Acta Colombiana de Psicología, 10, 107-115.

Quiroga-Baquero, L., Padilla, M. A., Ordoñez, S., \& Fonseca, L. (2016). Efectos de diferentes tipos de entrenamiento por modelado en tareas de igualación a la muestra. Revista Latinoamericana de Psicología, 48, 18-29. doi: 10.1016/j. rlp.2015.09.001. 
Ribes, E. (1997). The stimulus in behavior theory: Event or function? Revista Mexicana de Análisis de la Conducta, 23, 147-160. doi:10.5514/rmac.v23.i2.25218.

Ribes, E. (2000). Instructions, rules and abstraction: A misconstrued relation. Behavior and Philosophy, 28, 41-55.

Ribes, E., \& Rodríguez, M. E. (2001). Correspondence between instructions, performance, and self-descriptions in a conditional discrimination task: The effects of feedback and type of matching response. The Psychological Record, 51, 309333.

Ribes, E., \& Zaragoza, A. (2009). Efectos de las instrucciones y las descripciones con y sin criterio en la adquisición y transferencia de una discriminación condicional de segundo orden. Acta Comportamentalia, 17, 61-95.

Ribes, E., Moreno, D., \& Martínez, C. (1998). Second-order discrimination in humans: The roles of explicit instructions and constructed verbal responding. Behavioral Processes, 42, 1-18. doi: 10.1016/S0376-6357(97)00056-9.

Ribes, E., Torres, C., Barrera, J., \& Ramírez, L. (1995). Efectos de la variación modal de los estímulos en la adquisición y transferencia de una discriminación condicional en humanos adultos. Acta Comportamentalia, 3, 115-151.

Rodríguez-Pérez, M. E., Silva-Castillo, L. H., Bautista-Castro, L. R., \& Peña-Correal, T. E. (2015). Efectos de diferentes tipos de entrenamiento en el aprendizaje de una discriminación condicional. Acta Colombiana de Psicología, 18(1), 55-67. doi: 10.14718/ACP.2015.18.1.6.

Ruiz-Castañeda, D., \& Gómez-Becerra, M. (2016). Instructional control role in the study of verbal regulatory trends. Universitas Psychologica, 15(2), 135-152. doi: 10.11144/ Javeriana.upsy15-2.pcie.

Saunders, K., \& Williams, D. (1998). Stimulus-control procedures. En K. Lattal \& M. Perone (Eds.), Research Methods in Human Operant Behavior (pp. 193-228). New York: Plenum Press. doi: 10.1007/978-1-4899-1947-2.
Schlinger, H. (1993). Separating discriminative and functionaltering effects of verbal stimuli. The Behavior Analyst, 16, 9-23.

Serrano, M., García, G., \& López, A. (2006). Textos descriptivos de contingencia como estímulos selectores en igualación de la muestra con humanos. Acta Comportamentalia, 14, 131-143.

Serrano, M., García, G., \& López, A. (2008). Textos descriptivos de contingencia como instrucciones iniciales en tareas de igualación de la muestra. Acta Comportamentalia, 16, 333-346.

Serrano, M., García, G., \& López, A. (2009). Efectos de la retroalimentación para las respuestas de igualación correcta $\mathrm{o}$ incorrecta en la adquisición y transferencia de discriminaciones condicionales. Revista Mexicana de Análisis de la Conducta, 35, 113-134. doi: 10.5514/rmac.v35.i1.401.

Skinner, B. F. (1966). An operant analysis of problem solving. En B. Kleinmuntz (Ed.). Problem solving: Research, method and theory. (pp. 225-257). New York: John Wiley and Sons.

Stewart, I., \& McElwee, J. (2009). Relational responding and conditional discrimination procedures: An apparent inconsistency and clarification. The Behavior Analyst, 32, 309317.

Vaughan, M. (1989). Rule-governed behavior in behavior analysis: A theoretical and experimental history. En S. C. Hayes (Ed.), Rule-governed behavior: Cognition, contingencies, and instructional control (pp. 97-117). New York: Plenum.

Vega, M., \& Peña, T. E. (2008). Efecto de diferentes entrenamientos sobre el aprendizaje, transferencia y formulación de la regla en una tarea de igualación a la muestra de primer orden. Revista Colombiana de Psicología, 17, 115-127.

Zentall, T. R., Galizio, M., \& Critchfield, T. S. (2002). Categorization, concept learning, and behavior analysis: an introduction. Journal of Experimental Analysis of Behavior, 78, 237248. doi: 10.1901/jeab.2002.78-237. 